\title{
Comparing potential spatial access with self-reported travel times and cost analysis to haemodialysis facilities in North-eastern Iran
}

\author{
Behzad Kiani, ${ }^{1,2}$ Nasser Bagheri, ${ }^{3}$ Ahmad Tara, ${ }^{4}$ Benyamin Hoseini, ${ }^{5}$ Soheil Hashtarkhani, ${ }^{2}$ \\ Mahmood Tara ${ }^{2}$ \\ ${ }^{1}$ Vascular and Endovascular Surgery Research Center, Mashhad University of Medical Sciences, Mashhad, \\ Iran; ${ }^{2}$ Department of Medical Informatics, Faculty of Medicine, Mashhad University of Medical Sciences, \\ Mashhad, Iran; ${ }^{3}$ Department of Health Services Research and Policy, College of Health and Medicine, \\ Australian National University, Canberra, ACT, Australia; ${ }^{4}$ Department of Nephrology, Shahid Modarres \\ Hospital, Shahid Beheshti University of Medical Sciences, Tehran, Iran; ${ }^{5}$ Department of Health Information \\ Technology, Neyshabur University of Medical Sciences, Neyshabur, Iran
}

\begin{abstract}
End-stage renal disease patients regularly need haemodialysis three times a week. Their poor access to haemodialysis facilities is
\end{abstract}

Correspondence: Mahmood Tara, Department of Medical Informatics, Faculty of Medicine, Mashhad University of Medical Sciences, Mashhad, Iran.

Tel: +985138002428 - Fax: +985138002445

E-mail: Taram@mums.ac.ir

Acknowledgements: the authors would like to make special thanks to Dr. Mohsen Shahriari for his contribution to data gathering, as well as all the nurses who work in the haemodialysis facilities in northern Khorasan province of Iran.

Key words: Driving cost; Haemodialysis; Kriging; Spatial accessibility; Travel time; Iran.

Contributions: BK wrote the draft of manuscript, analysed data, and designed the study; NB and MT reviewed the multiple versions of manuscript and contributed on study design; NB revised the manuscript according to reviewers' comments. AT, BH and BK contributed on data gathering; SH computed travel costs of patients to dialysis facilities.

Conflict of interest: the authors declare no potential conflict of interest. Funding: n. 931059 from Mashhad University of Medical Sciences, Iran.

Ethical statement: this study was approved under the code IR.MUMS.RES.1393.756 by the Ethical Committee of Mashhad University of Medical Sciences in Mashhad, Khorasan Province, Iran. All patients completed a consent form for participating in the study.

Received for publication: 24 April 2018.

Revision received: 24 August 2018.

Accepted for publication: 24 August 2018.

(C) Copyright B. Kiani et al., 2018

Licensee PAGEPress, Italy

Geospatial Health 2018; 13:703

doi:10.4081/gh.2018.703

This article is distributed under the terms of the Creative Commons Attribution Noncommercial License (CC BY-NC 4.0) which permits any noncommercial use, distribution, and reproduction in any medium, provided the original author(s) and source are credited. significantly associated with a high mortality rate. The present cross-sectional study aimed to measure the potential spatial access to dialysis services at a small area level (census tract level) in North Khorasan Province, Iran. The patients were interviewed to obtain their travel information. The two-step floating catchment area (2SFCA) method was used to measure the spatial accessibility of patients to the dialysis centres. The capacity of the dialysis centre was defined as the number of active dialysis facilities in each centre and the haemodialysis patients in each area were considered as the users of dialysis services. The travel cost from each patient's residence to the haemodialysis facilities was visualized by the Kriging interpolation algorithm in the study area. Spatial accessibility to the dialysis centre was poor in the northern part of the study area. Fortunately, there were not many haemodialysis patients in that area. Patients' travel costs were high in the northern areas compared to the rest of study area. We observed a statistically significant reverse correlation between the self-reported travel time and computed spatial accessibility $(-0.570, \mathrm{P}$ value $<0.01$, two-tailed spearman test). This study supports the notion that the 2SFCA method could be associated with revealed access time to dialysis facilities, especially in low traffic and in flat areas such as northern Khorasan. The mapping of patients' distribution and interpolated travel cost to the haemodialysis facilities could help policymakers to allocate health resources to the areas where the need is greater.

\section{Introduction}

Chronic kidney disease is one of the major chronic conditions all over the globe. It gradually leads to kidney dysfunction resulting in irreversible stages such as end-stage renal disease (ESRD) in which the patient needs kidney transplantation or dialysis to compensate his/her renal dysfunction (Webster et al., 2017). Haemodialysis procedure is the most globally used method for ESRD patients to remove wastes from the blood (Foundation, 2015). To survive, haemodialysis patients need to travel from their house to dialysis centres at least three times a week (Garg et al., 2017). Previous studies have reported that patients' poor access to dialysis facilities is significantly associated with the high rate of mortality and lower quality of life (Moist et al., 2008; Rucker et $a l ., 2011)$. Recent advances in spatial analysis studies have been found to be practical in terms of investigating distribution appropriateness of the services required by dialysis patients due to their numerous references to these centres during the course of treat- 
ment (Stephens et al., 2013). Geographical information systems (GIS) provides spatial analyses by combining spatial and non-spatial data into one framework (Kiani et al., 2017). Such systems have been widely applied to health-related studies such as mapping of diseases and healthcare services (McLafferty, 2003). GIS for measuring access to dialysis facilities have drawn attention to be useful for research in this field in recent years (Hoseini et al., 2018). Access measurement has been conceptualized in numerous ways. McLafferty defines access as ease in receiving a service at a specified location and time (McLafferty, 2003). Access, however, has five main dimensions: accessibility, availability, accommodation, affordability and acceptability (Penchansky and Thomas, 1981). The accessibility and availability aspects are related to geographical factors, and are therefore called spatial access. Availability is expressed as the geographical distribution of the health services. Accessibility is defined by the physical distance between the health facility and the patient. In another classification, access is divided to potential and actual categories. Potential access is a proxy of the ability of individuals to use the services, but actual access expresses the actual use of the services.

In the present study, the two-step floating catchment area (2SFCA) method was used to measure the potential spatial access to dialysis services in North-Khorasan Province. To our knowledge, the effectiveness of this method has not yet been compared to actual access in dialysis context. Haemodialysis services were provided by dialysis centres using dialysis machines to treat patients. The Dialysis Guideline (Foundation, 2015) recommends a maximum distance of $40 \mathrm{~km}$ as the appropriate range for dialysis patients. We used this guideline to define the proper radial coverage distance for every dialysis centre. The primary objective of the present study was to investigate the association of potential spatial access measured by 2SFCA method with actual access indicated by self-reported travel times of dialysis patients. Additionally, we measured the cost of travel to haemodialysis facilities for each patient.

\section{Materials and Methods}

This study is built on previous studies for measuring accessibility to haemodialysis services in North Khorasan Province (Kiani et al., 2017a; Kiani et al., 2017b). We designed a cross-sectional study with participation of 165 haemodialysis patients. The study was conducted in five phases as follows.

\section{Phase 1: Data collection}

Two researchers visited all dialysis centres in North Khorasan and interviewed 165 participating patients. We obtained written consent from those patients who took part in the study. The interview information included patients' demographics, residence addresses, self-reported travel times and travel cost to dialysis centres. Moreover, we collected information on the number of active haemodialysis machines in each dialysis centre and their service profiles.

\section{Phase 2: Geocoding of patient addresses and haemodialysis centres}

Geocoding is a process in which physical addresses are converted to geographical coordinates (longitude and latitude). In this study, we geo-linked patients' residential addresses and services locations using Geocoding Web Service by Google (Googleplex, Mountain View, CA, USA), which provides users with 2,500 transaction per 24 hours free of charge. The research team developed a program based on Microsoft VB.NET programming language to communicate with this web service.

\section{Phase 3: Implementing the two-step floating catchment area method}

The 2SFCA method has been widely used by researchers in recent years to measure the spatial access to health services (Bagheri et al., 2008; McGrail, 2012). It includes a first step specifying catchment area for every service provider, followed by calculation of the number of service consumers for that service catchment and the ratio of capacity to service consumers for every service provider there. In a second step, an appropriate geographical area for receiving the service is specified, i.e. the population catchment area that includes all service consumers there, followed by specification of available service providers for each zone in that area. Finally, the sum of ratios of the service provider's capacity to the population covered by each service provider is calculated.

\section{Step 1}

Using the buffer analysis in ARC MAP10.3 software (ESRI, Redlands, CA, USA) a distance buffer of $40 \mathrm{~km}$ was set for each centre. The patient layer was spatially joined to each buffer area to calculate the number of service recipients in the given area $\left(P_{s}\right)$. Finally, the ratio of the capacity of active available haemodialysis machines $\left(C_{s}\right)$ to the number of haemodialysis patients $\left(P_{s}\right)$ was calculated. Subsequently, this data was joined to the data of the dialysis centre to determine the facility-to-population ratio for each haemodialysis service $(s)$ as shown by Eq. 1 .

$F P R_{s}=C_{s} / P_{s}$ Eq. 1

where $F P R_{s}$ is the facility to population ratio.

\section{Step 2}

First, the central point of each census tract was calculated as the population centre with regard to the haemodialysis patients, then a $40-\mathrm{km}$ buffer was calculated for each of these population centres. These buffers were spatially joined to the dialysis centre layers developed in the first step (including the facility-patient ratio) to calculate the index of the sum of haemodialysis machineto-patient ratios for each population centre buffer. This index, in fact, indicates the spatial access. Finally, the created buffer layers were joined to the census tract layers in the study area to specify the spatial access index for each census tract. We then visualized the access index at the census area level, and used Eq. 2 to compute accessibility index $(A)$ for each patient population $(i)$.

$A_{i}=\sum F P R_{s}$

Eq. 2

\section{Phase 4: Identifying haemodialysis patients' travel time, travel distance, routes to dialysis facilities, and correlation of self-reported travel time with potential spatial access}

The Google map web service (direction service) was used to calculate the patients' travel time and travel distance to dialysis facilities. Because traffic plays a major role during patients travel to the dialysis services, we estimated the travel time for morning 
and evening shift patients separately.

The dialysis patients' routes toward the dialysis centres were specified using Google's Direction Web Service. For each of the dialysis patients, the shortest path to the dialysis centre was obtained and then demonstrated on the map. Highly congested roads were shown in dense colouring for better visualization (Figure 1).

We used the non-parametric Spearman's correlation test (where $r=1$ means a perfect positive correlation and $r=-1$ a completely negative relationship) to measure the strength of association between self-reported travel time and potential spatial access for census tracts of the study area.

\section{Phase 5: Identification of travel cost paid by patients to dialysis facilities and interpolation of cost data}

We used self-reported cost of travel to the haemodialysis centres for those patients who did not travel by private car. However, for those patients who used their own cars to travel to the dialysis centres we calculated travel costs based on related factors in the literature (Barnes and Langworthy, 2003) due to unavailability of standardized driving costs in Iran. To estimate these travel costs, we extracted domestic values from the Saipa Pride company's website (http://www.saipayadak.org/470) which is the most popular automobile in Iran. We used Straight Line Depreciation considering five years as the useful life of a car for estimating the annual depreciation. In calculations, we also used a 20,000-km driving distance per year for each patient's car.

The Kriging algorithm was used for generating cost surface in the study area. Kriging is similar to Inverse Distance Weighting
(Kiani et al., 2017) in that it weights the surrounding measured values to derive a prediction for an unmeasured location (Wu et al., 2017). The method for interpolation is formed as a weighted sum of the data (Eq. 3):

$V\left(P_{0}\right)=\sum_{i=1}^{M} \lambda_{\mathrm{i}} V\left(P_{i}\right)$

Eq. 3

where $V\left(P_{i}\right)$ is the measured value at the $i^{\text {th }}$ location, $\lambda_{\mathrm{i}}$ an unknown weight for the measured value at the $i^{\text {th }}$ location, $P_{0}$ the prediction location, and $M$ the number of measured values.

\section{Results}

Figure 1 shows the patients' shortest routes from their houses to the dialysis centres across the province as mentioned above.

The distribution of the haemodialysis patients across the North Khorasan along with the buffer of the Euclidean distance of $40 \mathrm{~km}$ from each dialysis centre is shown in Figure 2. All geocoded addresses of patients for privacy issues were jittered. As seen in Figure 2, some areas, such as the northern part of the province, were not included in the $40-\mathrm{km}$ buffer of any of the haemodialysis centres.

Figure 3 shows the results of the 2SFCA method for measurement of the spatial access at census tract level in North Khorasan. As can be seen, the haemodialysis services are highly accessible in the western part of Shiravn City, while spatial accessibility is poor, even close to zero, in the North of the province. The area of Esfarayen City has also at a relatively good status in terms of spa-

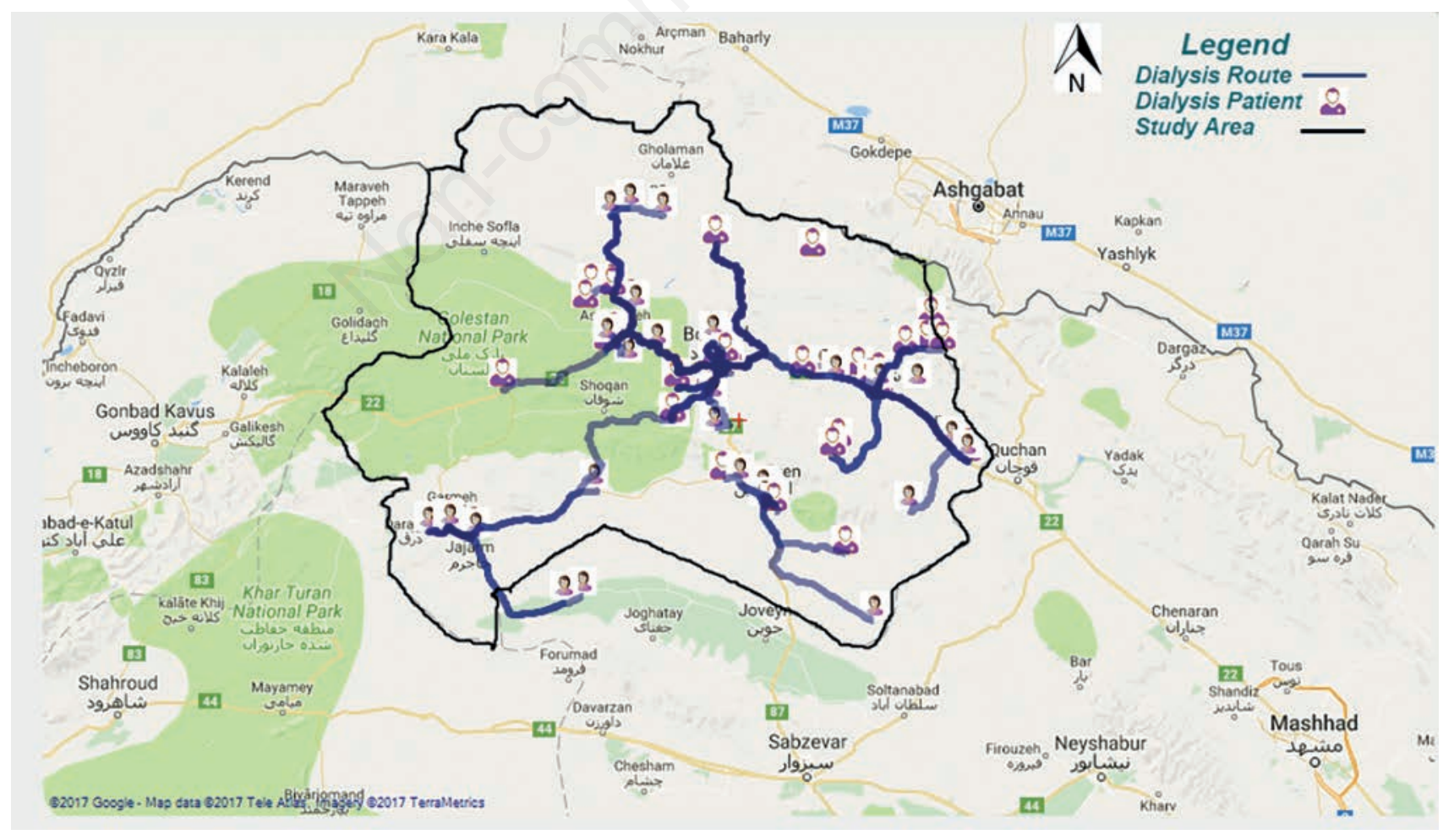

Figure 1. Dialysis patients' routes toward dialysis centres across North Khorasan Province, Iran. 
tial access. The self-reported travel time of patients to dialysis facilities shown in Figure 3 are generally good. However, there was a significant negative Spearman correlation $(-0.570, \mathrm{P}<0.01$, two-tailed) between the spatial accessibility and the self-reported travel time. As shown in Table 1, the calculated cost per km driving a Saipa Pride automobile is just above 5.5 US cents. Figure 4 shows the interpolated travel cost to dialysis facilities calculated by the Kriging algorithm for the study area. It shows that patients living in the northern part of the study area pay a higher cost to reach a dialysis service.

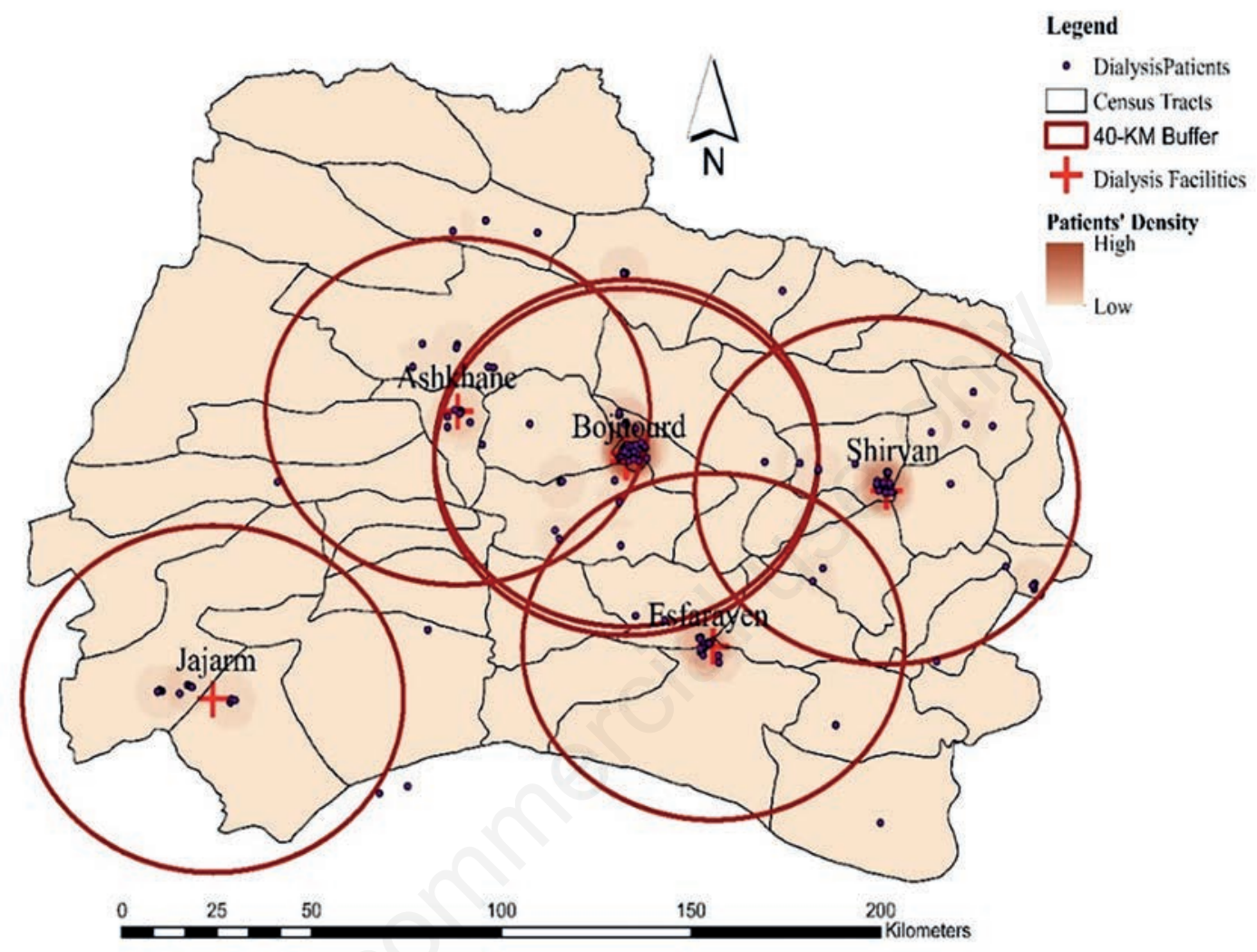

Figure 2. Geographical distribution (plus density) of haemodialysis patients in North Khorasan Province, Iran along with 40-km buffers of the haemodialysis centres.

Table 1. Cost of driving in Iran in 2017.*

\begin{tabular}{|c|c|c|c|c|c|}
\hline \multirow[t]{2}{*}{ Type of service } & \multirow{2}{*}{$\begin{array}{c}\text { Interval } \\
\text { (km) }\end{array}$} & \multicolumn{2}{|c|}{ Service cost } & \multicolumn{2}{|c|}{ Cost per km } \\
\hline & & Iran rial & US\$ & Iran rial & US\$ \\
\hline Fuel & 100 & 6400 & 1.60 & 64 & 1.60 \\
\hline Oil \& Filters & 5,000 & 85,000 & 21.25 & 17 & 0.43 \\
\hline Insurance & 100,000 & $3,991,000$ & 997.75 & 39.91 & 1.00 \\
\hline Tire change & 80,000 & 220,000 & 55.00 & 2.75 & 0.07 \\
\hline Battery/Cables & 100,000 & 25,000 & 62.50 & 2.5 & 0.06 \\
\hline Fan Belt & 40,000 & 105,000 & 26.25 & 2.625 & 0.07 \\
\hline Clutch parts & 60,000 & 160,000 & 40.00 & 2.66 & 0.07 \\
\hline Suspension & 80,000 & 140,000 & 35.00 & 1.75 & 0.04 \\
\hline Spark Plugs & 20,000 & 30,000 & 7.50 & 1.5 & 0.04 \\
\hline Accidents** & 20,000 & 250,000 & 62.5 & 12.5 & 0.31 \\
\hline Depreciation & 20,000 & $1,500,000$ & 375 & 75 & 1.88 \\
\hline Sum & & & & 222 & 5.56 \\
\hline
\end{tabular}

*Costs are based on Saipa Pride Car (http://www.saipayadak.org/470), the most popular automobile in Iran (http://bestsellingcarsblog.com/category/iran/). ${ }^{* * A c c o r d i n g ~ t o ~ i n f o r m a t i o n ~ f r o m ~ a n ~ i n s u r a n c e ~ e x p e r t . ~}$ http://vehicle.parsianinsurance.com/inquiry/start.html. All websites accessed 2017 on November 1, 2017. 


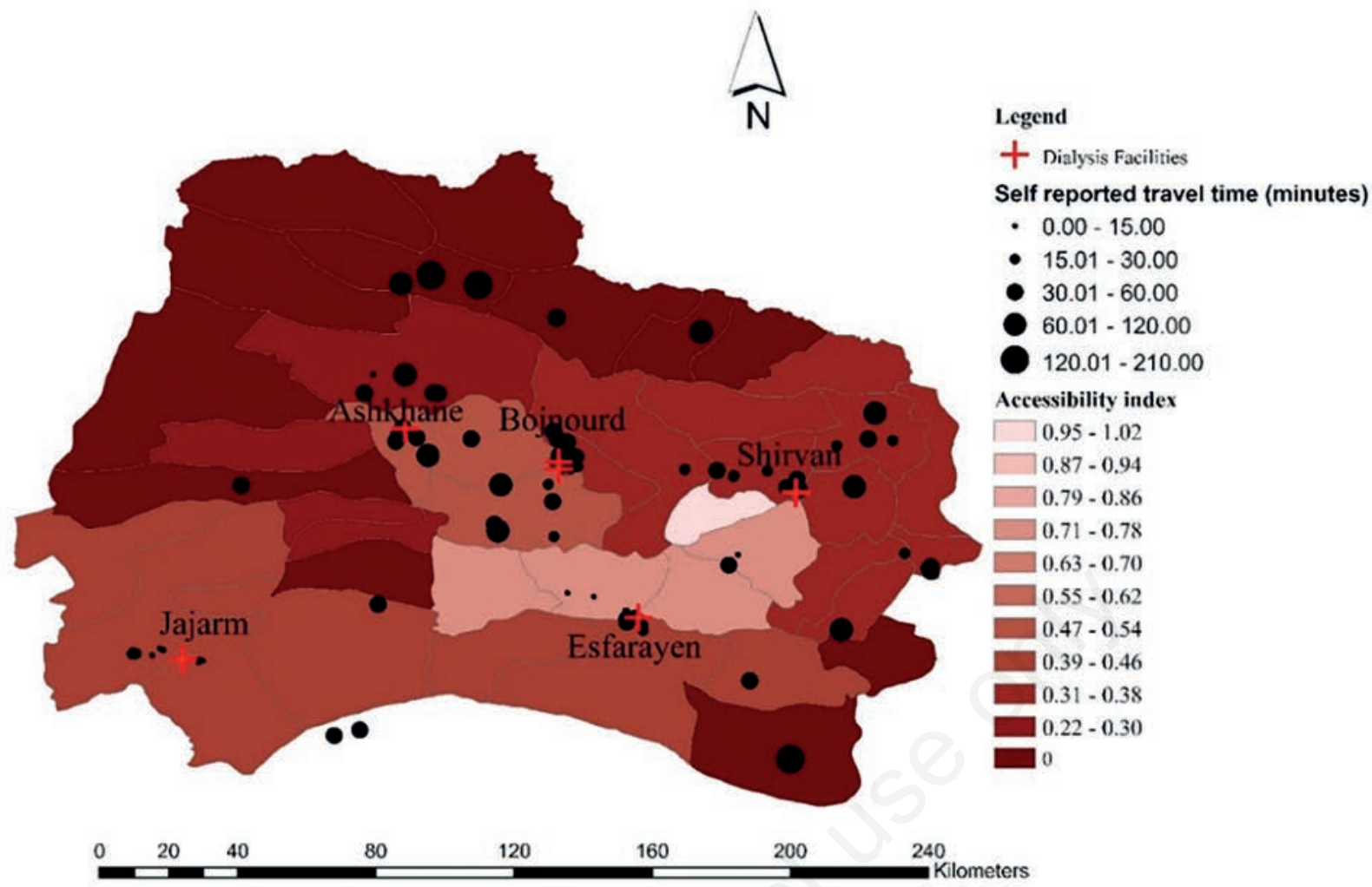

Figure 3. Spatial accessibility and patients' self-reported travel time to dialysis centres in North Khorasan Province, Iran.

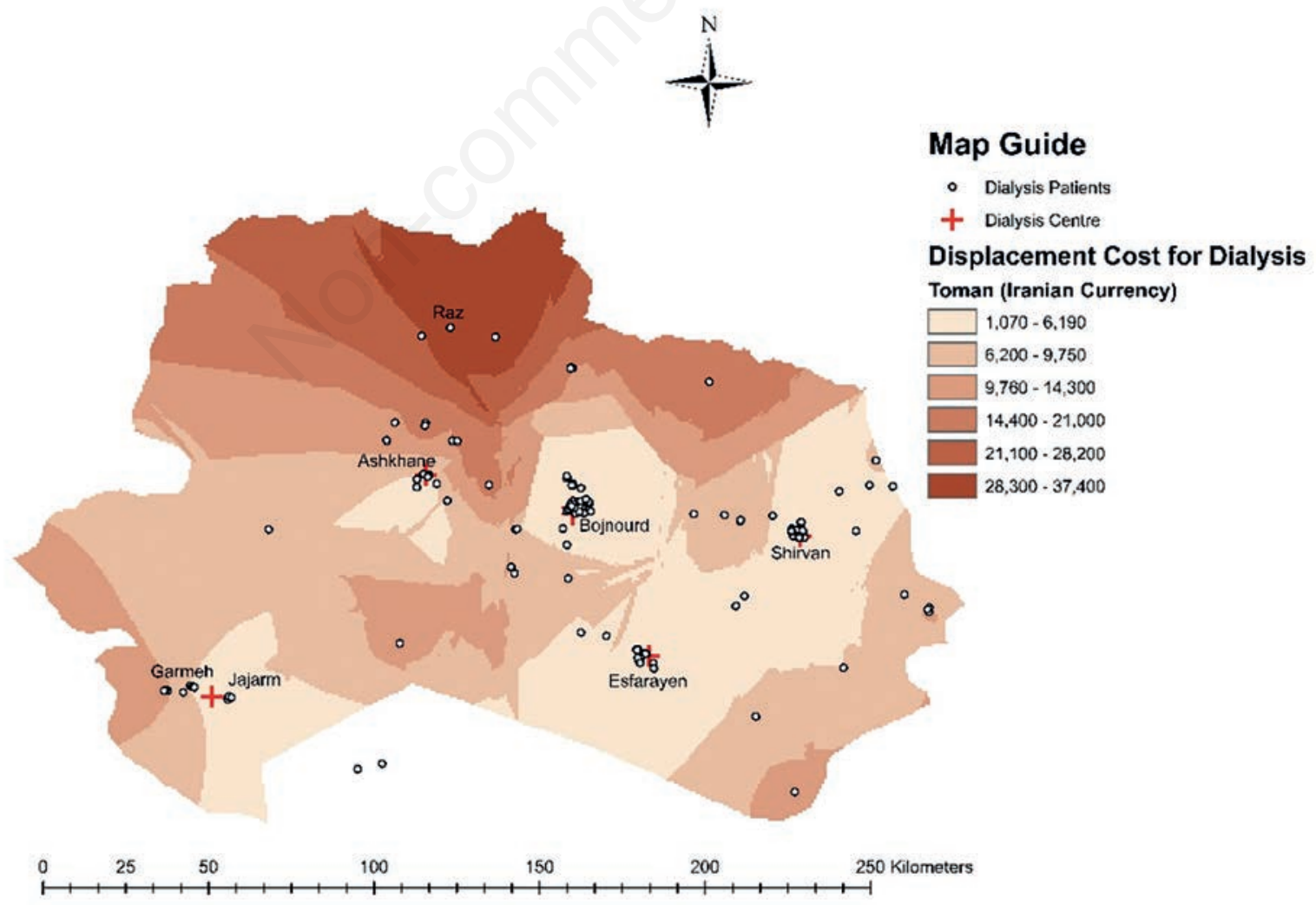

Figure 4. Interpolated travel cost to dialysis facilities calculated by Kriging algorithm. 


\section{Discussion}

Measuring the potential spatial access to dialysis facilities is one of the most fundamental analyses required for identifying underserved areas. In the present study, the potential spatial access to dialysis services across the North Khorasan Province in Iran was investigated. The small-area analysis using the 2SFCA method provided reliable local variations in spatial access pattern in accordance with McGrail's (2012) findings. As shown in Figure 1, the northern part of the province was found not to have an appropriate coverage of the dialysis centres. Although, currently only six dialysis patients live in this area, it must be emphasized that there are not a sufficient number of dialysis services there. The findings of this study may help policymakers to allocate appropriate services for enhancing patients' accessibility to dialysis services in the northern areas of the province. Considering the currently limited number of the dialysis patients in this region, adoption of one or more mobile haemodialysis station(s) is recommended. The 2SFCA method allows not only the study of supply itself but also the location of facilities, e.g., overlapping areas as low demand contributes to high access (Yang et al., 2006). As Figure 3 shows, the western part of Shirvan City has a high level of accessibility score with three facilities covering the haemodialysis population that only includes a low number of dialysis patients. The 2SFCA method also measures equity and equality of access to dialysis facilities (Richard et al., 2009; Vadrevu and Kanjilal, 2016), which means that the method considers the need for each area (number of patients) to compute the spatial access. The implication is that this approach enables policy makers to identify and better target haemodialysis services to areas where most needed. However, we are aware that even a perfect spatial access in a particular area would not necessarily lead to a better access in reality due to many other confounding factors (Casas et al., 2016). For example, several other studies have proved that the individual income levels (Scheer et al., 2003), gender (Neri and Kroll, 2003), ethnicity (Rodriguez et al., 2013), as well as public transportation availability (Mao and Nekorchuk, 2013) may also affect patients' revealed access. There was also a statistically significant association between self-reported travel times and the computed spatial access by 2SFCA method in this study (Figure 3) supporting the use of this method, which can then be considered a reliable method for multifactor access measurement. A study by Tshamba et al. (2014) showed that the cost of dialysis could be a negative, non-spatial factor for getting access to dialysis facilities. The dialysis procedure does not cost the patient anything because it is subsidized entirely by the government in Iran. However, patients would need to pay other costs such as transportation expenses and accommodation (Diamant et al., 2010; Mao and Nekorchuk, 2013). In accordance with these authors' findings, we discovered that patients located in the northern part of the study area which has low accessibility score, had to pay more cost to reach the haemodialysis facilities (Figure 4).

This study had some limitations. Due to lack of road network information, we used buffer analysis (Euclidian distance) to measure access to facility centres instead of driving time. Despite providing a simpler and faster method, the buffer analysis has lower precision than using driving time (McGrail, 2012). Buffer analysis can result in over-estimating the access to haemodialysis centres, especially in areas with heavy traffic and winding moun- tain roads. However, there are only a few such roads in our study area; thus it may not have affected our results. In this study, we used weighted population centroids for each census as a proxy for patient residence which may also have reduced the accuracy of access calculations. However, since we used the finer geographical scale as the unit of the spatial analysis, such effects were minimized.

\section{Conclusions}

The main contribution of this study is that the results of 2SFCA method was shown to be associated with revealed access time to dialysis facilities, especially in low traffic and flat areas such as Iran's northern Khorasan Province. The map of patient distribution this area and interpolated travel costs to the haemodialysis facilities is a powerful tool for policymakers to better allocate resources to the unmet areas.

\section{References}

Bagheri N, Benwell GL, PGDipSci M, 2008. Modelling Accessibility to Primary Health Care Using a Spatial Accessibility Index and a Need Index. Hawai 'I J Pub Health 1:14-27.

Barnes G, Langworthy P, 2003. The per-mile costs of operating automobiles and trucks. Retrieved from the University of Minnesota Digital Conservancy. Available from: http://hdl.handle.net/11299/909

Casas I, Delmelle E, Delmelle EC, 2016. Potential versus revealed access to care during a dengue fever outbreak. J Transport Health 4:18-29.

Diamant MJ, Harwood L, Movva S, Wilson B, Stitt L, Lindsay RM, Moist LM, 2010. A comparison of quality of life and travel-related factors between in-center and satellite-based hemodialysis patients. Clin J Am Soc Nephrol 5:268-74.

Foundation NK, 2015. KDOQI clinical practice guideline for hemodialysis adequacy: 2015 update. Am J Kidney Dis 66:884-930.

Garg AX, Suri RS, Eggers P, Finkelstein FO, Greene T, Kimmel PL, Kliger AS, Larive B, Lindsay RM, Pierratos A, 2017. Patients receiving frequent hemodialysis have better healthrelated quality of life compared to patients receiving conventional hemodialysis. Kidney Int 91:746-54.

Hoseini B, Bagheri N, Kiani B, Azizi A, Tabesh H, Tara M, 2018. Access to dialysis services: A systematic mapping review based on geographical information systems. Geospat Health 13:3-10.

Kiani B, Bagheri N, Tara A, Hoseini B, Tabesh H, Tara M, 2017 a. Revealed access to haemodialysis facilities in northeastern Iran: Factors that matter in rural and urban areas. Geospat Health 12:237-44.

Kiani B, Bagheri N, Tara A, Hoseini B, Tara M, 2017 b. Haemodialysis services in the northeastern region of Iran. Geospat Health 12:130-6.

Mao L, Nekorchuk D, 2013. Measuring spatial accessibility to healthcare for populations with multiple transportation modes. Health Place 24:115-22.

McGrail MR, 2012. Spatial accessibility of primary health care util- 
ising the two step floating catchment area method: an assessment of recent improvements. Int J Health Geogr 11: 50-61.

McLafferty SL, 2003. GIS and health care. Ann Rev Public Health 24:25-42.

Moist LM, Bragg-Gresham JL, Pisoni RL, Saran R, Akiba T, Jacobson SH, Fukuhara S, Mapes DL, Rayner HC, Saito A, Port FK, 2008. Travel time to dialysis as a predictor of healthrelated quality of life, adherence, and mortality: the Dialysis Outcomes and Practice Patterns Study (DOPPS). Am J Kidney Dis 51:641-50.

Neri MT, Kroll T, 2003. Understanding the consequences of access barriers to health care: experiences of adults with disabilities. Disabil Rehabil 25:85-96.

Penchansky R, Thomas JW, 1981. The concept of access: definition and relationship to consumer satisfaction. Med Care 19:127-40.

Richard JB, Aldigier JC, Le Mignot L, Glaudet F, Ben Said M, Landais P, 2009. Equity of accessibility to dialysis facilities. Stud Health Technol Inform 150:777-81.

Rodriguez RA, Hotchkiss JR, O'Hare AM, 2013. Geographic information systems and chronic kidney disease: racial disparities, rural residence and forecasting. J Nephrol 26: 3-15.

Rucker D, Hemmelgarn BR, Lin M, Manns BJ, Klarenbach SW, Ayyalasomayajula B, James MT, Bello A, Gordon D, Jindal KK, Tonelli M, 2011. Quality of care and mortality are worse in chronic kidney disease patients living in remote areas. Kidney Int 79:210-7.
Scheer J, Kroll T, Neri MT, Beatty P, 2003. Access Barriers for Persons with Disabilities The Consumer's Perspective. J Disability Policy Studies 13:221-230.

Stephens JM, Brotherton S, Dunning SC, Emerson LC, Gilbertson DT, Harrison DJ, Kochevar JJ, McClellan AC, McClellan WM, Wan S, Gitlin M, 2013. Geographic disparities in patient travel for dialysis in the United States. J Rural Health 29:33948.

Tshamba HM, Van Caillie D, Nawej FN, Kapend FM, Kaj FM, Yav GD, Nawej PT, 2014. Risk of death and the economic accessibility at the dialysis therapy for the renal insufficient patients in Lubumbashi city, Democratic Republic of Congo. Pan African Med J 19:61-8.

Vadrevu L, Kanjilal B, 2016. Measuring spatial equity and access to maternal health services using enhanced two step floating catchment area method (E2SFCA)-a case study of the Indian Sundarbans. Int J Equity Health 15:87-98.

Webster AC, Nagler EV, Morton RL, Masson P, 2017. Chronic kidney disease. The Lancet 389:1238-52.

Wu H, Wang X, Xue M, Xue M, Wu C, Lu Q, Ding Z, Xv X, Lin J, 2017. Spatial characteristics and the epidemiology of human infections with avian influenza A (H7N9) virus in five waves from 2013 to 2017 in Zhejiang Province, China. PloS One 12:e0180763.

Yang DH, Goerge R, Mullner R, 2006. Comparing GIS-based methods of measuring spatial accessibility to health services. $\mathrm{J}$ Med Syst 30:23-32. 